\title{
Automated Registration of Ultrasound with $C T$ Images: Application to Computer Assisted Prostate Radiotherapy and Orthopedics
}

\author{
Gelu Ionescu, PhD., Stéphane Lavallée, PhD., Jacques Demongeot, MD., PhD.
}

TIMC Laboratory, University Joseph Fourier, Grenoble France

gelu.ionescu@imag.fr

\begin{abstract}
Ultrasound $(U S)$ imaging could potentially play a major role in the field of Computer Assisted Surgery (CAS). For doctors and surgeons to make full use of CAS tools in planning and executing surgical operations, they also need user-friendly automatic software based on fast, precise and reliable registration algorithms. The main goal of this paper is to take advantage of the segmentation/registration duality to extract the relevant information from $U S$ images. This information will allow the precise and automatic registration of anatomical structures contained in the pre-operative model and of intra-operative data contained in $U S$ images. The result of registration will be further used to guide a computerassisted intervention such as orthopedics or radiotherapy.
\end{abstract}

\section{Introduction}

Among the various imaging techniques available, $2 D$ echography is becoming more and more important, both for the diagnostic purposes and registration in CAS applications. In this paper, we will consider the latter case, with the specific instance of Computer Assisted Orthopedics Applications [1,2] and patient positioning in prostate radiotherapy [3]. In spite of the problems inherent to $U S$ imaging such as low signal-to-noise ratio and the fact that the images depend on the angle and the texture of the target anatomy, this modality has become very popular among practitioners mainly because of its safety, low cost and non-invasiveness.

Since external radiotherapy makes use of the properties of $X$-rays to destroy live tissue, the precise localization of the tumor is extremely important in order to avoid the destruction of neighboring healthy tissues. As described in details in [4], in the context of isocentric technique applied to radiotherapy, the procedure for repositioning must ensure that the center of the tumor localized by the practitioner in the pre-operative model, coincides with the center of rotation of the radiation device. For the intra-operative data acquisition, we are interested particularly in the transpubic way to realize the $U S$ examination of the prostate. The goal of the registration is to realize an automatic orientation of the external $X$ - ray beams according to a predetermined dosimetry plan. The results presented here were obtained on patients. In orthopedic applications, a 
high precision is also required in order to respect the planning established by the surgeon. Our aim is either to place a screw in a pedicle vertebra [2], or to insert percutaneously screws in the sacro-iliac bone [1,5]. In both cases, the goal is to realize a minimally invasive operation. To demonstrate the feasibility of the method, we worked, for the first application, on a plastic vertebra immersed in water, for the second application tests were performed on a cadaver.

In this paper, we present an automatic, reliable and precise method that can be used in soft tissues (e.g. abdominal cavity) as well as in hard tissues (e.g. bone, cartilage). The medical imagings we focus mainly on, are computed tomography $(C T)$ and $U S$ imaging. The former is used to build the pre-operative $3 D$ model and the latter to acquire the $3 D$ intra-operative data volume.

\section{Related Work}

The general problem of registration between a set of $3 D$ data points and a $3 D$ model was treated in $[6,7]$. In this paper, we consider a $3 D$ model made from a set of $C T$ slices. This approach has been discussed in $[2,5]$ for various applications. The methods known in the literature is based on the segmentation of the surface $S$ of an anatomical structure on $C T$ images. This is followed by a manual segmentation of pieces of contours corresponding to this same structure on $U S$ images. Each $U S$ image being located in the intra-operative reference frame by using an external optical localization device, one obtains an image of points belonging to the surface of the reference structure. The rigid registration of $3 D$ points with the surface $S$ starting from an initial position is described in various papers $[7,8,9]$. Unfortunately none of the existing segmentation procedure of $U S$ images is automatic, precise and robust. Here we propose a method featuring these characteristics [10].

Grey-Level Approach The most obvious method, as often used in registration, would be to register directly $C T$ images with $U S$ images without going through segmentation. For images originating from the same modality, one could use methods based on maximization of correlation, confinement tree [11]. For images of different nature, optimization based on mutual information could be used [12]. For a given initial registration $T_{0}$, for each $U S$ image in a given position, one can calculate the slice corresponding to the echographical plane in the volume of $C T$ images but the calculation of reformatted slices is very time consuming [10]. For this reason an approach based on the use of contours seemed preferable.

Contour Approach As a starting point, the $3 D$ model is assumed to be both segmented and labeled. To achieve this it is of course possible to use semiinteractive procedures since the model is constructed before the operation. In general, one segments contours on $2 D$ slices. These are then interpolated to create a homogeneous $3 D$ surface using the shape-based interpolation algorithm described in $[13,10]$. Although many registration/3D labeling procedures are described in the literature, those based on elastic registration of a model (Atlas 
like) seem the most promising ones [14]. The segmentation/registration duality was discussed previously in different context. Hamadeh [15] proposed to register the $3 D$ model of a segmented vertebra on $C T$ images with intra-operative $X-$ ray of the same vertebra. Mangin [16] proposed a two-step registration of Positron Emission Tomography and $C T$ images of the brain while recently Geraud [17] proposed a progressive approach using Atlas-type models to segment the Magnetic Resonance images of the brain. Our approach, as in the above cited works, is based on the segmentation/registration duality, its specificities are that we rely on $U S$ imaging and that we propose an original mechanism for segmentation, guided by the model. As in the method based on gray-levels, we propose to generate pseudo $U S$ images, however, we strictly limit ourselves to the contours of structures segmented in the model. This is equivalent to considering only the specular reflection and to neglect all other phenomena. This approach is detailed in the following sections.

\section{Methods}

The method we have proposed consists of three stages, the first consists of a low-level segmentation of a sequence of $U S$ images. The second stage exploits the segmentation/registration duality to extract relevant contours obtained in the previous step. The last step consists of the final rigid registration of these relevant contours and the pre-operative model.

\subsection{Intra-operative Data Acquisition}

Images are taken from an $U S$ imager. The $U S$ probe is localized in $3 D$ space by an optical localizer. The $U S$ image is calibrated according to a technique described in [10]. In this way, the position of an image pixel is known in $3 D$ space with a precision in the range of localizer accuracy (about $1 \mathrm{~mm}$ ).

\subsection{Low-Level Segmentation}

By low-level segmentation, we mean classical image segmentation techniques based mainly on linear filtering or mathematical morphology. At the end of this stage, the images are generally over-segmented (presence of false positives) [18] and the contours are not labeled. In this work, we choose the watershed as the segmentation technique. The main advantages of watershed segmentation relative to methods based on filtering [19] are the following: the watershed always contains some segments placed correctly on the real contours, the resulting contours are closed, detection of multiple junctions is very insensitive to noise and the algorithm is based on the modulus of the gradient $\|\nabla I\|$ of the image (the direction of the gradient is ignored).

To eliminate undesirable effects produced by the speckle on the complexity of the watershed, the original image is filtered by a recursive Canny-Deriche filter [20]. The width of this filter is chosen in an empirical way but it remains 
unchanged for the processing of the whole sequence of images. Furthermore, we observed that the filter width does not change significantly from one patient to the next. For the test-patient used for the prostatic application, the parameter for the Canny-Deriche filter is $\alpha=0.2$. The result of this first step presented in Fig. 1.e shows two false watershed segments going through the bladder/prostate structure and the right iliac bone. One notes immediately that the prostate is well isolated which demonstrates that the use of watershed is completely realistic. At this stage, the processing is specific to the structure that needs to be detected.

\subsection{High-Level Segmentation}

At this stage, we assume the availability of a set of $M$ initial attitudes $T_{i}(i=1, M)$, relating the echographical data and the $C T$ model. Each initial attitude $T_{i}$ is applied to each of the $N$ echo slices. One then obtains $M$ possible intersections between each echo plane and the dense cluster of points representing the surface of the model. In order to increase the resolution for the exploration of the volume of the model, to each initial attitude $T_{i}$, one associates six additional transformations $\Delta T_{j}(j=0,6)$ which slightly displace the image plane as presented in Fig. 2.a. In this scenario, we voluntarily neglected the displacements inside the image plane since these could be compensated by the $2 D / 2 D$ elastic registration described in this section.

For each combination $\left(T_{i}, \Delta T_{j}\right)$, we keep the model points which lie within the thickness of the $U S$ beam $(\simeq 2 \mathrm{~mm})$. We then perform an elastic multi-level $2 D / 2 D$ registration between the simulated echo slice and the real echo slice. As described hereinafter, we minimize an expression of the form:

$$
\varepsilon=\sum_{i=1}^{N} \operatorname{dist}_{2 D}\left(Q_{j}, T_{p}\left(P_{i}\right)\right)_{i=1, M}
$$

where $P_{i}$ is the set of $N$ simulated echo points, $Q_{j}$ is the set of $M$ real echo points and $T_{p}$ is the elastic $2 D$ transformation relative to the parameter vector $p$ we are looking for.

In order to give to the registration process the necessary elasticity, we associate to the image a regular network, the nodes of which can be individually adjusted. A displacement vector $\left(V_{x}, V_{y}\right)^{\tau}$ is associated to each node as presented in Fig. 2.b. The global transformation $T_{p}$ we are looking for is parameterized by the vector $p$ :

$$
p=\left(\alpha, t_{x}, t_{y} ;\left(V_{x}, V_{y}\right)_{0,0}^{\tau}, \cdots,\left(V_{x}, V_{y}\right)_{i, j}^{\tau}, \cdots,\left(V_{x}, V_{y}\right)_{I_{x}, I_{y}}^{\tau}\right)^{\tau}
$$

where $\tau$ means transposition, $\left(I_{x}, I_{y}\right)$ is the network dimension, $\left(\alpha, t_{x}, t_{y}\right)^{\tau}$ is the purely rigid component and $\left(\left(V_{x}, V_{y}\right)_{0,0}^{\tau}, \cdots,\left(V_{x}, V_{y}\right)_{i, j}^{\tau}, \cdots,\left(V_{x}, V_{y}\right)_{I_{x}, I_{y}}^{\tau}\right)^{\tau}$ is the purely elastic component of the transformation $T_{p}$. To determine the vector $p$, we have developed a nonlinear iterative optimization algorithm based on the 

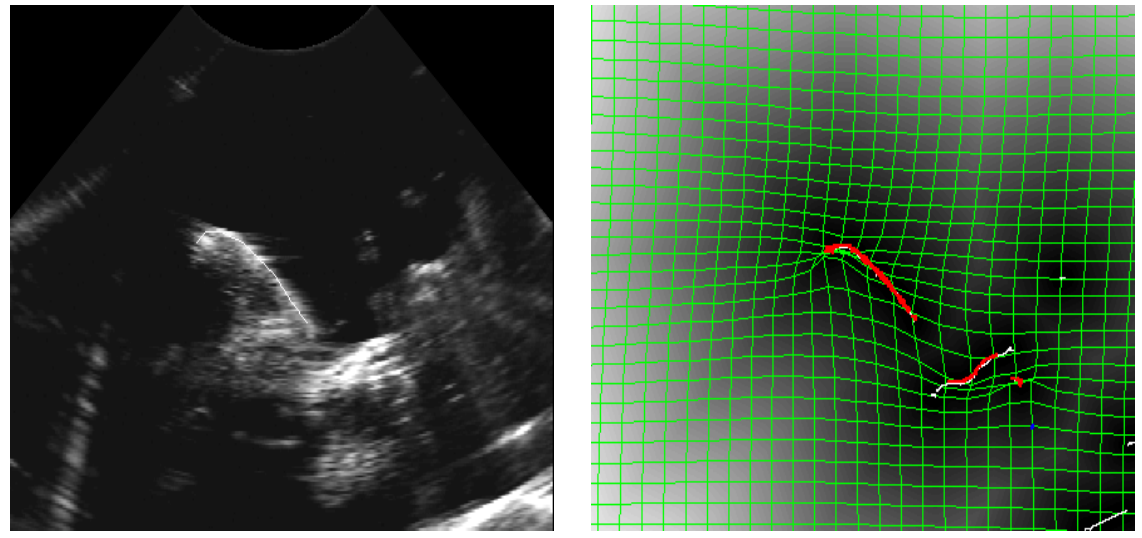

(a)

(b)
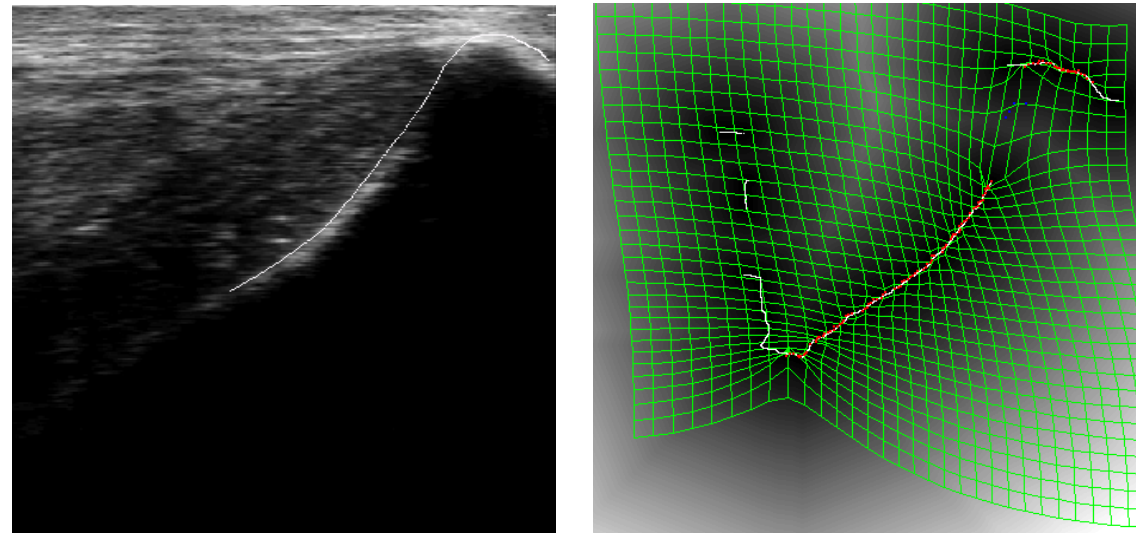

(c)

(d)
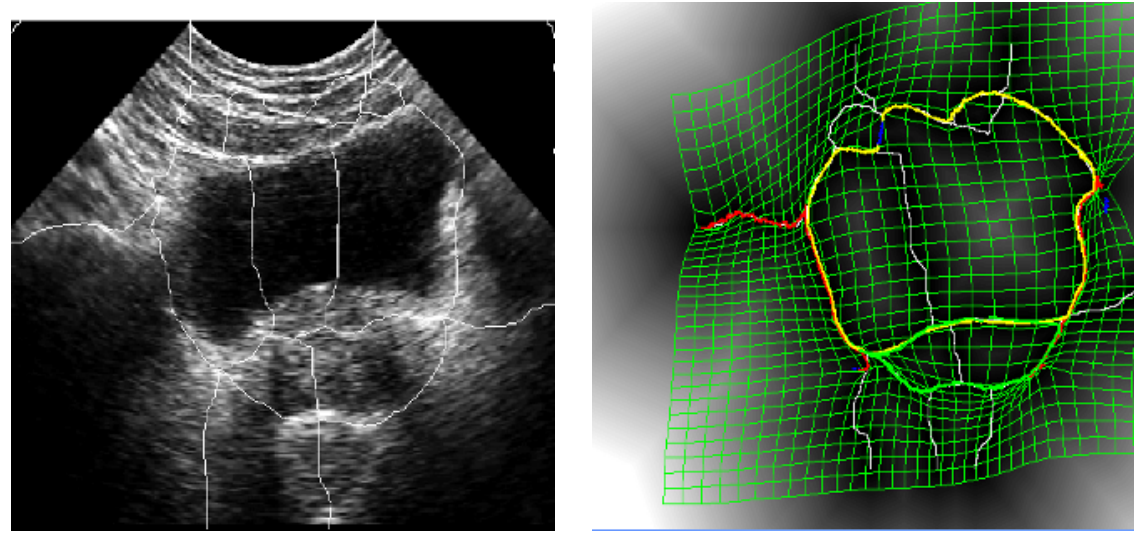

(e)

Fig. 1. Typical $U S$ images (left) and $2 D / 2 D$ elastic registration (right). (a-b) Vertebra. (c-d) Sacro-iliac region. (e-f) Bladder and prostate. 

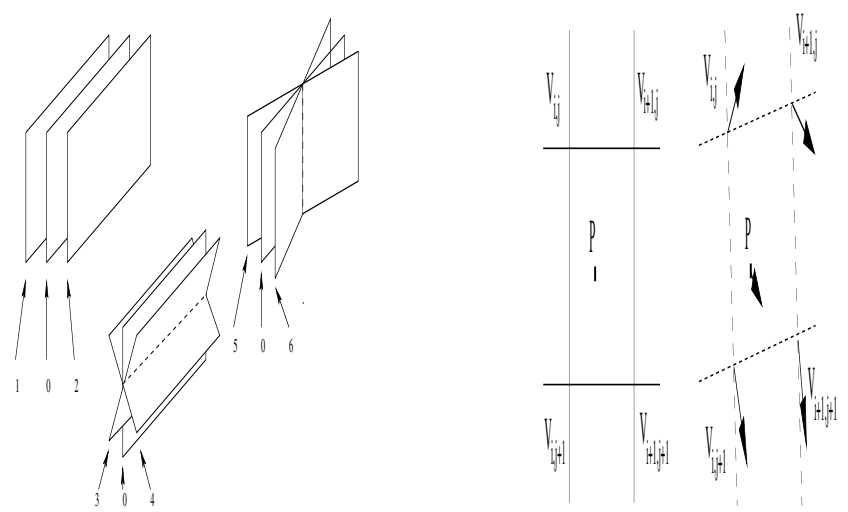

(a)

Fig. 2. (a) Additional transformations associated to initial attitude $T_{i}$. (b) Elastic $2 D$ transformation.

minimization of the following objective function:

$$
\begin{aligned}
\varepsilon= & \frac{1}{N} \sum_{i=1}^{N}\left\|Q_{j}-T_{p}\left(P_{i}\right)\right\|^{2}+\rho_{1} \frac{1}{I_{x} \times I_{y}} \sum_{(i, j)=[0,0]}^{\left[I_{x}, I_{y}\right]}\left\|V_{i, j}\right\|^{2}+ \\
& \rho_{2} \frac{1}{I_{x} \times I_{y}}\left(\sum_{(i, j)=[0,0]}^{\left(I_{x}, I_{y}\right]}\left\|V_{i+1, j}-V_{i, j}\right\|^{2}+\sum_{(i, j)=[0,0]}^{\left[I_{x}, I_{y}\right)}\left\|V_{i, j, 1}-V_{i, j}\right\|^{2}\right)
\end{aligned}
$$

where the first term represents the distance from the transformed point $P_{i}$ to the nearest point $Q_{j}$, the second term regularizes the dimension of the vectors $V_{i, j}$ associated with the $(i, j)$ network node and the third term regularizes the similarity of vectors associated with neighboring nodes.

To speed up the computation of distances, we use the $2 D$ chamfer distance map for the set of real points $Q_{j}$. The minimization method used is based on the calculation of gradient and uses the following iterative mechanism:

$$
\begin{aligned}
p_{k}^{t+1} & =p_{k}^{t}-\lambda \nabla \varepsilon_{k} \\
\delta p_{k} & =-\lambda \nabla \varepsilon_{k}
\end{aligned}
$$

where $\lambda$ is a dimension less scale factor and $\nabla \varepsilon_{k}$ is the gradient of the objective function expressed in (3) relative to the component $k$ of the vector $p$.

Convergence Criteria The criteria for stopping the iterative procedure are related to the energy and the gradient:

1. $\frac{\varepsilon_{t+1}^{2}-\varepsilon_{t}^{2}}{\varepsilon_{t}^{2}} \leq T_{1}$; the relative energy gain between two successive successful iterations must be below a reasonable threshold $T_{1}$. 
2. $N_{\text {iterations }}$; after $N_{\text {iterations }}$ successive unsuccessful iterations.

3. $\left(I_{x}, I_{y}\right)$; after the convergence using the previously defined criteria, the network resolution can be increased and the minimization starts again.

Retaining the Best $\left(T_{i}, \Delta T_{j}\right)$ Combination In the case of hard tissues, the choice of the best $\left(T_{i}, \Delta T_{j}\right)$ combination relies on a likelihood principle. Consequently, we construct an objective discriminating function based on the first term of (3) and on the number of points $P_{i}$ of the model. Among many possibilities, the solution retained is based on the Student test $t$ in order to compare variances. We have adopted the solution proposed in [21] where the original data are transformed in the absolute values of the deviation of each distance relative to the average value using the relation:

$$
\begin{aligned}
& t=\left(\bar{x}_{1}-\bar{x}_{2}\right) s \sqrt{\frac{1}{n_{1}}-\frac{1}{n_{2}}} \\
& s=\left(\left(n_{1}-1\right) s_{1}^{2}+\left(n_{2}-1\right) s_{2}^{2}\right) /\left(n_{1}+n_{2}-2\right)
\end{aligned}
$$

where $n_{1,2}$ is the sample size, $\bar{x}_{1,2}$ is the sample mean value, $s_{1,2}$ is the sample variance and $s$ is the global variance. The value $t_{k, l}$ obtained from expression (6) is used as a dissimilarity measure for the couple $\left[(T, \Delta T)_{k},(T, \Delta T)_{l}\right]$. A symmetric matrix $T=\left[t_{k, l}\right] ; k, l \in[1, N \cdot M]$ is then constructed. An objective function $f_{t}(l)=\sum_{k=1}^{N \cdot M} t_{k, l}$ is obtained by summing over the columns of the matrix $T$. The decision mechanism is based on minimizing $f_{t}(l)$. Consequently, the optimal $(T, \Delta T)_{\text {opt }}$ combination corresponds to the relation $\left(f_{t}\right)_{o p t}=\min _{l=1, N \cdot M} f_{t}(l)$.

In the case of soft tissues, one cannot defend the use of maximum likelihood as an objective discriminatory function. For instance it is very easy to register elastically two transversal slices through the bladder. Taking into account the preceding observation, we propose a solution adapted to all soft tissues based on two criteria, a quantitative one followed by a qualitative one: 1. Establish a measure that determines how well the points of the model fit the intra-operative data. To achieve this, we compute a distance map based on the new coordinates of the deformed model points taken after the $2 D / 2 D$ elastic registration. From this distance map, we obtain the distance of each intra-operative data point relative to the model. The average value of all these distances, $\bar{d}_{i, j}$, defines the objective measure characterizing the $\left(T_{i}, \Delta T_{j}\right)$ combination. The best combination being the one with an uniform distribution of the points of the deformed model as compared with intra-operative data. With a minimization procedure one obtains $(T, \Delta T)_{o p t}=\min _{l=1, N \cdot M}\left(\bar{d}_{i, j}\right)_{l}$. 2. In problematic situations when none of the $\left(T_{i}, \Delta T_{j}\right)$ combinations represent a realistic situation (the model points are not well-distributed) this image will be marked and eliminated from the final $3 D / 3 D$ registration process. The decision criteria are the following: the model points must cover at least $50 \%$ of the data and in the case of the prostate, this area must necessarily cover the base of the bladder. After the choice of the best $(T, \Delta T)_{\text {opt }}$ combination, another step of $2 D / 2 D$ elastic registration will be necessary. This time, intra-operative data are represented by the watershed 
while pre-operative model is represented by the set of anatomic structure of the region of interest (i.e. the bone, the bladder and the prostate). The results are presented in Fig. 1.

\subsection{Rigid Registration}

Choice of Segments For this step, we had to choose between two possibilities: isolate all segments of the watershed pointed by the model or retain the new coordinates of the points of the model. To show that both possibilities are realistic, we used the first one in orthopedic applications and the second one in radiotherapy applications.

A final $3 D / 3 D$ registration step is necessary to match the points previously extracted and labeled on $U S$ images with the surface of the $C T$ model. For that purpose, one could use elastic registration [22], but both the prostate (for radiotherapy) and the bones (for orthopedics) are considered as rigid bodies. Therefore, standard $3 D$ surface registration techniques were used to estimate the transformation between $C T$ model and the patient coordinate system in the local region of interest [23].

\section{Results}

In order to validate our technique, we compared the final results obtained after the rigid $3 D / 3 D$ registration with either those obtained from a manual segmentation of the prostate or, in the case of the orthopedic application, with manual digitalization of the bone surface with a finger probe localized in $3 D$ space. All numerical results are presented in the following table where we display the errors obtained both on rotations $\varepsilon_{\max }^{\alpha}$ expressed in degrees and on translations $\varepsilon_{\max }^{t}$ expressed in $\mathrm{mm}$. In the last column, the same results are presented in terms of Rodriguez's rotation and translation.

\begin{tabular}{|c||c|c|c|}
\hline & $\varepsilon_{\max }^{\alpha}[\mathrm{deg}]$ & $\varepsilon_{\max }^{t}[\mathrm{~mm}]$ & Rodriguez \\
\hline prostate & $(-1.23,1.30,0.75)$ & $(0.19,-0.67,-2.58)$ & $\left(2.67^{\circ}, 2.07 \mathrm{~mm}\right)$ \\
\hline vertebra & $(1.90,2.05,-0.98)$ & $(-0.15,-0.52,0.87)$ & $\left(1.23^{\circ}, 1.70 \mathrm{~mm}\right)$ \\
\hline bassin & $(-2.93,1.13,-1.83)$ & $(-0.39,0.12,-1.02)$ & $\left(1.14^{\circ}, 1.91 \mathrm{~mm}\right)$ \\
\hline
\end{tabular}

In Fig. 3, we present the repositioning of a typical $U S$ image in $3 D$ space of the $C T$ per-operative model. Fig. 3.a displays the superposition of the model on the spinal process of the vertebra, Fig. 3.b presents a superposition with the $U S$ image representing the sacrum and Fig. 3.c displays an US image of the bladder and the prostate.

\section{Conclusion}

In this paper, we have presented automatic and robust algorithms necessary for registration between a $3 D C T$ model and a set of 2.5D US images. Essentially, these algorithms use methods of low-level segmentation as described in 

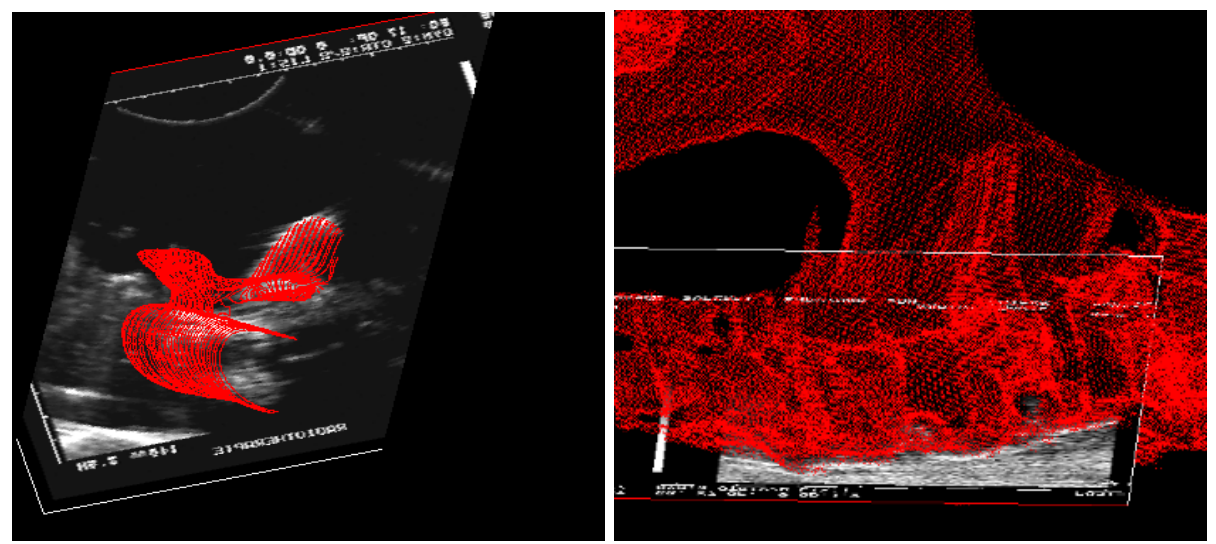

(a)

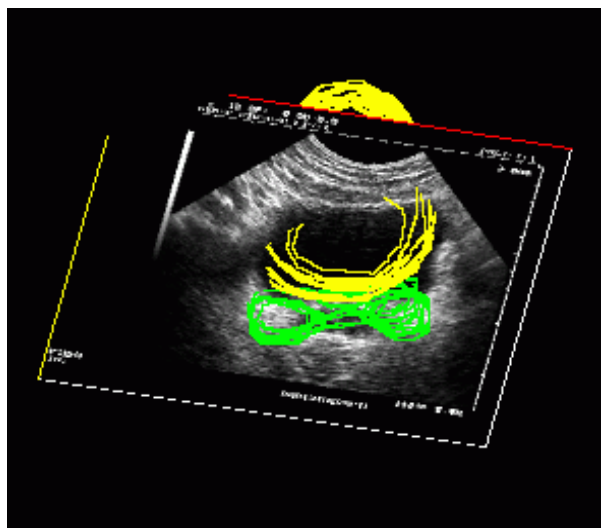

(c)

Fig. 3. Results: (a) Vertebra registration. (b) Sacrum registration. (c) Bladder and prostate registration

paragraph $\S 3.2$ and high-level segmentation by labeling as described in $\S 3.3$. To emphasize the generality of our approach, each application has been analyzed in the following framework: description of clinical objectives, presentation of specific pre-processing, labeling by elastic $2 D / 2 D$ registration, choice of segments and presentation of results. In all this work, we paid special attention to the precision and we could demonstrate that the maximum errors are about $2 \mathrm{~mm}$ and $2^{0}$ which is compatible with most of applications. While the orthopedic application chosen clearly deals with high-risk regions, in the case of the prostate, the risk is to irradiate healthy organs. Considering the important risks inherent to an intervention on the spine or in the sacral region, we have tested our method in vitro. The preliminary results obtained in the automatization of the segmentation process are very encouraging. It is clear that the result of a minimization 
procedure generally used in matching depends strongly on the precision of the initial attitude $T_{0}$, this also affects the global computing time. To improve on this, we hope to find a procedure that estimates an initial attitude $T_{0}$ as close as possible to the final attitude for each application. We plan also to correct the $U S$ images, in term of the velocity of $U S$. This will influence the precision of the results specially in the radiotheray applications. This method is generic and it could be easily applied to other organs such as liver and kidney.

\section{References}

1. Tonetti J. et al, Percutaneous iliosacral screw placement using image guided techniques, CORR special issue, pp. 103-110, 1998. 768, 769

2. Merloz P. et al, Pedicle screw placement using image guided techniques, CORR special issue, pp. 39-48, 1998. 768, 769

3. Troccaz J. et al, Conformal external radiotherapy pf prostatic carcinoma: requirements and experimental results, Radiother. Oncol., no. 29, pp. 176-183, 1993. 768

4. Vassal P., Fusion d'images multi-modales pour la radioth. conformationnelle: appli. au repos. du patient, Ph.D. Thesis TIMC-IMAG, Grenoble University, 1998. 768

5. Carrat L.et al, Treatment of pelvic ring fractures: percutaneous computer assisted iliosacral screwing, MICCAI'98-Proceedings, pp. 84-91, 1998. 769

6. Lavallée S. et al, Computer integrated surgery and therapy, C. Roux and J.L. Coatrieux ed., Contemp. Persp. in 3D Biomed. Im. IOS Press, pp. 239-310, 1997. 769

7. Maintz A. and Viergever M.A., A survey of medical image registration, Medical Image Analysis, vol. 2, no. 1, pp. 1-36, 1998. 769

8. Pelizzari C.A. et al, Accurate $3 \mathrm{D}$ registration of $\mathrm{CT}, \mathrm{PET}$ and/or MR images of brain, L. Comput. Assist. Tomo., no. 13, pp. 20-26,1989. 769

9. Lavallée S. et al, Matching 3D smooth surf. with their 2D proj. using 3D dist. maps, SPIE vol. 1570 Geom. Models in Computer Vision - San Diego, pp 322-336, 1991. 769

10. Ionescu G., Segm. et recalage d'images échographiques par utilisation de conaissances physio. et morpho., Ph.D. Thesis TIMC-IMAG, Grenoble University, 1998. 769,770

11. Mattes J. and Demongeot J., Dynamic confinement, classification and imaging, New techn. of classific., Lecture Notes in Comput. Sci., Springer Verlag, 1998. 769

12. Wells W.M. et al, Multimodal volume registration by minimisation of mutual information, Medical Robotics and CAS Wiley New York, pp 55-62, 1995. 769

13. Grevera G.J. and Udupa J.K., Shape-based interpolation of multidimensional greylevel images, IEEE Trans. Medical Imaging, vol. 15, no. 6, pp. 881-891, Dec. 1996. 769

14. Bittar E., Modèles déformables surfaciques, implicites et volumiques, pour l'imagerie médicale, Ph.D. Thesis TIMC-IMAG, Grenoble University, 1998. 770

15. Hamadeh A., Une approche unifiée pour la segm. el la mise en corresp. 3D/2D d'images multi-modales, Ph.D. Thesis TIMC-IMAG, Grenoble University, 1997. 770

16. Mangin J., Mise en correspondance d'images médicales 3D multi-modalités pour la corrélation anatomo-fonctionnelle cérébrale, Ph.D. Thesis ENST Paris, 1995. 770 
17. Géraud T., Segmentation des structures internes du cerveau en IRM 3D, Ph.D. Thesis TLCOM Paris, 1998. 770

18. Maes F., Segmentation and registration of multimodal medical images, Ph.D. Thesis ISBN 90-5682-135-0 D/1998/7515/37, 1998. 770

19. Najman L. and Schmitt M., Geodesic saliency of watershed contours and hierarchical segmentation, IEEE Trans. PAMI, vol. 18, no. 12, pp. 1163-1173, Dec. 1996. 770

20. Deriche R., Fast algorithms for low-level vision, IEEE Trans. PAMI, vol. 12, no. 1, pp. 78-87, Jan. 1990. 770

21. Manly B., Statistical methods, Chapman and Hall, 1989. 774

22. Szeliski R. et al, Matching 3-D anatomical surfaces with non-rigid deformations using octree-splines, Intern. Journ. of Comp. Vis., 18(2), pp. 171-186, 1996. 775

23. Lavallée S. et al, Recovering the pos. and orient. of free-form obj. from image contours using 3D dist. maps, IEEE Trans. PAMI, vol. 17, no. 4, pp. 378-390, 1995. 775 